\title{
NON-ADHERENCE IN SENIORS WITH DEMENTIA - A SERIOUS PROBLEM OF ROUTINE CLINICAL PRACTICE
}

\author{
Jan Lužnýt,2, Kateřina Ivanová' Lubica Juričkováa
}

Department of Psychogeriatry, Mental Hospital Kroměříž, Czech Republic'; Department of Social Medicine and Public Health, Faculty of Medicine, Palacky University, Olomouc, Czech Republic ${ }^{2}$

Summary: Background: Non-adherence to treatment in seniors with dementia is a frequent and potentially dangerous phenomenon in routine clinical practice which might lead to the inappropriate treatment of a patient, including the risk of intoxication. There might be different causes of non-adherence in patients with dementia: memory impairment, sensory disturbances, limitations in mobility, economical reasons limiting access to health care and medication. Non-adherence leads to serious clinical consequences as well as being a challenge for public health. Aim: to estimate prevalence of non-adherence in seniors with dementia and to study correlation between cognitive decline and non-adherence. Subjects and Methods: Prospective study, analyzing medical records of seniors with dementia admitted to the inpatient psychogeriatric ward in the Kromeriz mental hospital from January 2010 to January 2011. Cognitive decline measured by MMSE, prevalence of Non-adherence to treatment and reasons for patient Non-adherence were studied. Results: Non-adherence to any treatment was detected in $31.3 \%$ of seniors; memory impairment was the most common cause of non-adherence to treatment. Conclusion: In conclusion, non-adherence to treatment in the studied group of seniors with dementia correlates with the severity of cognitive impairment - a higher cognitive decline correlates with a higher risk of non-adherence to treatment.

Key words: Seniors; Non-adherence; Dementia; Memory impairment; Visual disturbances; Drug interactions

\section{Introduction}

Adherence to treatment means a patient's ability to follow the instructions given for the prescribed treatments $(1$, 2 ). These instructions might be given in an oral or written form by a doctor, nursing staff or other health care professional. The quality of the patient-doctor alliance can reflect adherence to medication by a patient (3). Non-adherence to treatment means that the medication is not used by a patient at all, or it is used in a wrong way (dosage, frequency of use of prescribed medication). Non-adherence to treatment is a serious problem - it may lead to the misinterpretation of estimating the effects of a prescribed therapy by physicians, followed by an elevation in dosage or changing of therapy for a less suitable one, moreover, it can endanger a non-adherent patient by causing adverse side effects or intoxication by giving an increased dosage of therapy which has little or no clinical effect. Medication with a narrow therapeutic dosage range (range between effective and toxic dosage) is of particular clinical importance (4). Medication non-adherence had a significantly negative impact on treatment response, highlighting the importance of adherence for achieving a satisfactory treatment outcome (5). In the same way, non-adherence to treatment is a significant economic burden put on health care systems $(6,7,8)$. Pharmacotherapy is the third most expensive issue in health care expenditures in the Czech Republic (9). If the medication is not used at all, or if the medication is used in a wrong way, then pharmacotherapy is just a waste of money. Non-adherence to treatment is a stressful event for prescribing physicians and it may lead to uncertainty about diagnosis or pharmacotherapy in a patient (6). It is difficult to estimate the prevalence of non-adherence to treatment - non-adherent patients are usually not able to reveal their non-adherence to physicians because of many reasons - they feel ashamed, they fear the physician's anger as a result of non-adherence, or very simply, they have problems in communication with their physician (2). All the factors mentioned above are also risk factors for non-adherence (3). Patients with mental disorders are a frail patient's group as for non-adherence - because of psychiatric reasons or unacceptable side effects of therapy (10). Non-adherence to treatment has been most often studied in patients with schizophrenia (11) and affective disorders $(12,13)$ because of the clinical, economic and social impact of these serious psychiatric conditions. Non-adherence in patients with schizophrenia is estimated in a wide range between $30-70 \%$ of patients $(10,14)$. The communicative skills of a patient's physician, psycho-education of a patient, intellectually intact patients and family support seem to be important protective factors for non-adherence $(15,16,17)$ and the best way for handling non-adherence to treatment $(2,18)$. A comprehensive review of the litera- 
ture on the measurement, correlates, and health outcomes of medication adherence among community-dwelling older adults was done by Vik et al. - they found there is little empirical data to support a simple systematic descriptor of the non-adherent patient. The inconsistencies across studies may be attributable, in part, to the inherent difficulties involved in the measurement of a behavioural risk factor such as non-adherence (19).

\section{Subjects and Methods}

Aim of the study. We tried to estimate prevalence of non-adherence in seniors with dementia and to study correlation between cognitive decline and non-adherence. We studied patient's reasons for non-adherence and complications due to non-adherence.

Design. A prospective study analyzing the medical records of seniors admitted to the inpatient psychogeriatric ward in the Kromeriz mental hospital from January 2010 to January 2011 has been designed.

Definitions. Non-adherence was defined as any violation of the instructions given for prescribed treatments by a patient who has been objectively reported by caregivers or relatives (lack of medication use, altered frequency of medication use, alteration in dosage). This fact is mandatorily recorded in our medical records during the admissions procedure and assists us in the further management of treatment. Inappropriate combination of drugs was defined as any combination of drugs that has differed from outpatient medical records known to us at the moment of admission to our facility (researchers had full access to the outpatient records belonging to GP's). This information was also confirmed by caregivers or relatives. Overdosing or under dosing due to non-adherence was defined as any dosage of a drug which was prescribed by a physician but which was taken in higher or lower dose by a patient. While inappropriate combination of drugs referred to type of drug, overdosing or under dosing due to non-adherence referred to dose of drug. Relapse or worsening of mental disorder was defined as a worsening of mental status, objectively perceived by caregivers or relatives, leading to a search for outpatient psychiatric services. Relapse or worsening of somatic disorder was defined as a worsening of somatic status, objectively perceived by caregivers or relatives, leading to a search for an outpatient health professional service (general practitioner, internist etc.). Sub-intoxication, intoxication or adverse effects were defined as a presence of symptoms developing as a consequence of non-adherence to treatment. These symptoms were mentioned in the outpatient medical records known to us at the moment of admission to our facility and they were verified in the AISLP database (Automated Information System of Registered Drugs) according to the definitions of sub-intoxication, intoxication or adverse effects to a certain drug profile. Reasons for patient non-adherence were asked during the admissions procedure in cases of non-adherent patients and noted in medical records.

Screening tools. The Mini-Mental State examination (MMSE) was used as a screening tool for estimating the severity of dementia - MMSE subdivides patients into groups of patients with mild, moderate and severe dementia, with regard to the prevalence of non-adherence.

Including and excluding criteria. Including criteria: informed consent of a patient aged 65 and more, first hospitalization of a senior due to dementia of any type and severity, diagnosis of dementia. Excluding criteria: patients under 65 years of age, mental diagnosis different from dementia, patient in terminal state.

Data analysis. Descriptive statistics was used for the evaluation of results; a statistical analysis tested a possible correlation between the severity of dementia (moderate, middle and severe dementia) and the prevalence of non-adherence to treatment (Kruskal-Wallis Test, Mann-Whitney test) at a 0.05 level of significance.

Ethical considerations. All the subjects agreed to participate in the research (informed consent). No ethical hazard due to the study is known to the author of the paper.

\section{Results}

In total, 855 elderly patients were hospitalized for dementia in the psychogeriatric ward in the Kromeriz mental hospital from January 2010 to January 2011. The average age was 71.2 years (standard deviation 5.6 years); among this group of seniors 387 patients were men (45.3\%), 468 patients were women (54.7\%). The average MMSE score was 15.8 (standard deviation 6.3). Non-adherence to any type of medication was detected in $31.3 \%$ of hospitalized seniors with dementia. Non-adherence to psychiatric medication appeared in $38.1 \%$ of all non-adherent patients $(\mathrm{n}=$ 268), non-adherence to somatic medication was detected in $61.9 \%$ of all non-adherent patients $(n=268)$. More detailed differences among the groups of patients with mild, moderate and severe dementia are shown in Table 1.

Regardless of the severity of dementia, the patients' primary reasons for non-adherence to treatment were different - the most frequent primary reason was memory impairment, when the patient had forgotten if the prescribed drug was taken or not. This cause of non-adherence to treatment was detected in $38.4 \%$ of non-adherent patients. Sensory impairment (especially visual disturbances) caused non-adherence to treatment in $14.2 \%$ of non-adherent patients. Economic reasons of non-adherence were surprisingly detected in $9.3 \%$ of non-adherent patients - for these patients drugs were too expensive. The existence of side effects of any type caused by therapy was detected in $18.7 \%$ of cases of non-adherence. Distrust in therapy has been referred to in $7.1 \%$ of non-adherent patients, followed by distrust of physicians, reported by $4.5 \%$ of non-adherent patients (Table 2 ). 
Tab. 1: Prevalence of non-adherence to treatment.

\begin{tabular}{|c|c|c|c|c|c|c|c|c|}
\hline & \multicolumn{2}{|c|}{ Mild dementia* } & \multicolumn{2}{|c|}{$\begin{array}{c}\text { Moderate } \\
\text { dementia }^{* *}\end{array}$} & \multicolumn{2}{|c|}{$\begin{array}{c}\text { Severe } \\
\text { dementia }^{* * *}\end{array}$} & \multicolumn{2}{|c|}{ TOTAL } \\
\hline & Abs. & Relat. & Abs. & Relat. & Abs. & Relat. & ABS. & RELAT. \\
\hline & (n) & $(\%)$ & (n) & $(\%)$ & (n) & $(\%)$ & (n) & $(\%)$ \\
\hline Patients & 422 & 49.4 & 275 & 32.2 & 158 & 18.4 & 855 & 100.0 \\
\hline Non-adherence to any medication & 77 & 28.7 & 86 & 32.1 & 105 & 39.2 & 268 & 31.3 \\
\hline Non-adherence to psychiatric medication & 21 & 20.6 & 40 & 39.2 & 41 & 40.2 & 102 & $38.3^{+}$ \\
\hline Non-adherence to somatic medication & 56 & 79.4 & 46 & 60.8 & 64 & 59.8 & 166 & $61.9^{+}$ \\
\hline
\end{tabular}

Tab. 2: Patient's reasons for non-adherence (regardless to the severity of dementia).

\begin{tabular}{|l|c|c|}
\hline & $\begin{array}{c}\text { Absolutely } \\
\text { (n) }\end{array}$ & $\begin{array}{c}\text { Relatively } \\
(\%)\end{array}$ \\
\hline Non-adherent patients & $\mathbf{2 6 8}$ & $\mathbf{1 0 0 . 0}$ \\
\hline Primary memory impairment & 103 & 38.4 \\
\hline Primary adverse effects of therapy & 50 & 18.7 \\
\hline Primary sensory impairment & 38 & 14.2 \\
\hline Primary economic reasons & 25 & 9.3 \\
\hline Primary distrust in therapy & 19 & 7.1 \\
\hline Primary distrust of physician & 12 & 4.5 \\
\hline Other reasons & 21 & 7.8 \\
\hline
\end{tabular}

\begin{tabular}{|l|c|c|}
\hline Subintoxication or intoxication & 19 & 7.1 \\
\hline $\begin{array}{l}\text { Other complications of Non- } \\
\text { adherence }\end{array}$ & 5 & 1.9 \\
\hline No complication of Non-adherence & 12 & 4.4 \\
\hline
\end{tabular}

The Mann-Whitney test focused on finding possible differences between the level of cognitive impairment (in the group of mild, moderate and severe dementia) and the prevalence of non-adherence to treatment. Significant differences in the prevalence of non-adherence regarding the level of cognitive impairment were found (level of cognitive impairment correlates with prevalence of non-adherence to treatment) - see table below (Table 4).

Tab. 4: Prevalence of non-adherence and severity of dementia statistical analysis

Regardless of the severity of dementia, the study focused on the possible occurrence of complications due to non-adherence to treatment. Relapse or worsening of a mental disorder was detected in $28.7 \%$ of non-adherent patients, while relapse or worsening of a somatic disorder was detected in $44.8 \%$ of non-adherent patients. Potentially dangerous findings revealed in the study were the adverse effects resulting from non-adherence to prescribed medication (observed in $13.1 \%$ of non-adherent patients) and cases of sub-intoxication or intoxication caused by non-adherence to treatment (observed in $7.1 \%$ of non-adherent patients). No complication of non-adherence to treatment was detected in only $4.4 \%$ of non-adherent patients (Table 3 ).

Tab. 3: Complications due to non-adherence

\begin{tabular}{|l|c|c|}
\hline & $\begin{array}{c}\text { Absolutely } \\
\text { (n) }\end{array}$ & $\begin{array}{c}\text { Relatively } \\
\text { (\%) }\end{array}$ \\
\hline Non-adherent patients & $\mathbf{2 6 8}$ & $\mathbf{1 0 0 . 0}$ \\
\hline $\begin{array}{l}\text { Relapse/worsening of mental } \\
\text { disorder }\end{array}$ & 77 & 28.7 \\
\hline $\begin{array}{l}\text { Relapse/worsening of somatic } \\
\text { disorder }\end{array}$ & 120 & 44.8 \\
\hline Adverse effects & 35 & 13.1 \\
\hline
\end{tabular}

\begin{tabular}{|l|c|}
\hline Compared groups of patients & Significance* \\
\hline Mild vs. moderate dementia & 0.017 \\
\hline Mild vs. severe dementia & $<0.001$ \\
\hline Moderate vs. severe dementia & $<0.001$ \\
\hline
\end{tabular}

* Mann-Whitney test, p-value 0.05

\section{Discussion}

An estimation of the prevalence of non-adherence to treatment in the population of Czech seniors with dementia is missing when compared to data from world literature (17, $20,21)$. The findings indicate that the processes of medication management differ according to the level of dementia. A number of corresponding facilitators and barriers to medication management were identified. Medication management in the early stages of dementia is characterized by the patients' desire to maintain independence, denial of issues or disease, and a refusal to take medications due to a feeling of angry. In late stage dementia, older adults often refuse medications because of delusional or suspicious thinking, which results in caregivers assuming responsibility for managing their medications (18). The prospective study has revealed 
a high prevalence of non-adherence to treatment in group of seniors hospitalized for dementia (31.3\% of patients with dementia in total). This finding is consistent with the results of other researchers $(22,23)$. Kardas (24) has found an even higher prevalence in non-adherence to treatment in cognitive impaired seniors (83.8\% non-adherent patients in his study).

It seems to be important to further subdivide groups of patients according to the level of cognitive impairment (mild, moderate and severe dementia), The Mini-Mental State Examination was the screening tool used to do this, as employed in similar studies of other authors (25). In this way, an estimation of the prevalence of non-adherence in relation to the severity of dementia can be revealed (20). In the descriptive study, the most common cases of non-adherence to treatment are the under dosing of prescribed medication (53.0\%), using an unprescribed combination of drugs $(31.0 \%)$ and overdosing of prescribed medication (16.0\%). All these possibilities can endanger the patient due to insufficient treatment, intoxication or atypical side effects of such a combination (4). In this study, the majority of non-adherent patients suffered some of the adverse conditions mentioned above (relapse or worsening of mental disorder, relapse or worsening of somatic disorder, side effects resulting from non-adherence to prescribed medication, sub-intoxication or intoxication). Only $4.4 \%$ of patients had no complications due to non-adherence to treatment, which is consistent with other researchers (21, 23). In studying the possible risk factors of non-adherence to treatment, memory impairment was detected as the most common risk factor $(38.4 \%$ cases of non-adherence in the studied group of patients). This result is consistent with literature (19). In accordance with Ganguli (20), non-adherence to treatment in the studied groups of seniors with dementia correlates with the severity of cognitive impairment.

Limitations of the study. Researchers had full access to the outpatient records belonging to GPs, but usually not records belonging to specialists. Some data could be missing because records belonging to GPs may not have been complete. We tried to verify all the recorded data with caregivers or information from relatives. However, data can be biased due to the varying quality of GP records. Non-adherence either to psychiatric medication or somatic medication was followed, but not cases of simultaneous non-adherence to both types of medication. The patients' primary reasons for non-adherence were studied separately, but not in possible combination as can happen in real life conditions. The validity of informed consent signed by patients with severe dementia may be discussed. No gender aspect of non-adherence was taken into account.

\section{Conclusion}

Non-adherence to treatment in seniors with dementia seems to be a common and serious problem in medical practice. Non-adherence to medication can lead to inappropriate therapy of a disease, with a risk of developing serious side effects, including intoxication. From the public health point of view, non-adherence increases economic expenditures in an inappropriate way; therefore it is also an interesting topic for public health. A higher cognitive decline in a patient means a higher risk of non-adherence to treatment. Early detection of dementia in seniors, establishing a good therapeutic alliance between patients and physicians, and supervising caregivers and their assistance in drug administration to seniors with severe dementia can be the most effective preventive measures to avoid the complications of non-adherence. Assistance in medication administration is necessary quite early in severe dementia, and supervision is advisable even in patients with moderate dementia.

\section{Acknowledgements}

No economical profit or ethical hazard is known to the author of this study.

\section{References}

1. Levensky ER, O'Donohue WT. Eds. Promoting treatment adherence: A Practical handbook for health care providers. 1st ed. California: Sage Publications Inc. 2006

2. Haynes RB, McKibbon KA, Kanani R. Systematic review of randomised trials of interventions to assist patients to follow prescriptions for medications. The Lancet 1996; 348(9024): 383-386.

3. Ascher-Swanum H, Zhu B, Faries D, et al. Prospective study of risk factors for Non-adherence with antipsychotic medication in the treatment of schizophrenia. The Journal of Clinical Psychiatry 2006; 67(7): 1114-23.

4. Hynie S. Farmakologie v kostce [Pharmacology at glance]. Textbook of pharmacology in Czech language. 1st ed. Praha: Triton 1998.

5. Lindenmayer JP, Liu-Seifert H, Kulkarni PM, et al. Medication non-adherence and treatment outcome in patients with schizophrenia or schizoaffective disorder with suboptimal prior response. The Journal of Clinical Psychiatry 2009; 70(7): 990-996.

6. Gilmer TP, Dolder CR, Lacro JP, et al. Adherence to treatment with antipsychotic medication and health care costs among medic aid beneficiaries with schizophrenia. Am J Psychiatry 2004; 161: 692-699.

7. Safran DG, Neuman P, Schoen C, et al. Prescription drug coverage and seniors: findings from a 2003 national survey. Health Affairs (Project Hope). Suppl Web Exclusives 2005; W5-152-W5-166.

8. Neuman P, Kitchman Strollo M, et al. Medicare prescription drug benefit progress report: findings from a 2006 national survey of seniors. Health Affairs 2006; 26(5): w630-w643.

9. Gladkij I. Management ve zdravotnictví. [Management in Health Care]. Textbook in Czech language. Brno: Computer Press 2003.

10. Schimmelmann BG, Paulus S, Schacht M, et al. Subjective distress related to side effects and subjective well-being in first admitted adolescents with early-onset psychosis treated with atypical antipsychotics.Journal of Child and Adolescent Psychopharmacology 2005; 15(2): 249-58.

11. Lacro JP, Dunn LB, Dolder CR, et al. Prevalence of and risk factors for medication Non-adherence in patients with schizophrenia: a comprehensive review of recent literature. The Journal of Clinical Psychiatry 2002; 63(10): 892-909.

12. Lingam R, Scott J. Treatment non-adherence in affective disorders. Acta Psychiatrica Scandinavica 2002; 105(3): 164-172.

13. Sajatovic M, Davies M, Hrouda DR. Enhancement of Treatment Adherence Among Patients With Bipolar Disorder. Psychiatric Services 2004; 55: 264-269.

14. Sernyak MJ, Dausey D, Desai R, et al. Prescribers' Non-adherence to treatment guidelines for schizophrenia when prescribing neuroleptics. Psychiatric Services 2003; 54(2): 246-8.

15. Swanson A, Pantalon M, Cohen K. Motivational interviewing and treatment adherence among psychiatric and dually diagnosed patients. Journal of Nervous \& Mental Disease 1999; 187(10): 630-635.

16. Sajatovic M, Sohaj O, Youngstrom E, et al. Treatment adherence in individuals with rapid cycling bipolar disorder: results from a clinical-trial setting. Journal of Clinical Psychopharmacology 2007; 27(4): 412-414.

17. Wilson IB, Schoen C, Neuman P, et al. Physician-patient communication about prescription medication non-adherence: a 50-state study of America's seniors. Journal of General Internal Medicine 2007; 22(1): 6-12.

18. Kaasalainen S, Dolovich L, Papaioannou A, et al. The process of medication management for older adults with dementia. Journal of Nursing and Healthcare of Chronic Illness 2011; 3(4): 407-418. 
19. Vik SA, Maxwell CJ, Hogan DB. Measurement, correlates, and health outcomes of medication adherence among seniors. Ann Pharmacother 2004; 38(2): 303-312.

23. Mansur N, Weiss A, Hoffman A, et al. Continuity and adherence to long-term drug treatment by geriatric patients after hospital discharge: a prospective cohort study. Drugs Aging 2008; 25(10): 861-70.

20. Ganguli M, Yangchun MS, Rodriguez E, et al. Discrepancies in information provided to primary care physicians by patients with and without dementia: the Steel Valley seniors survey. American Journal of Geriatric Psychiatry 2006; 14(5): $446-455$.

21. Arlt S, Lindner R, Rösler A, et al. Adherence to medication in patients with dementia: predictors and strategies for improvement. Drugs \& Aging 2008; 25(12): 1033-1047.
22. Barat I, Andreasen F, Damsgaard EM. Drug therapy in the elderly: what doctors believe and patients actually do. Br J Clin Pharmacol 2001; 51(6): 615-622.

23. Thiruchselvam T, Naglie G, Moineddin R, et al. Risk factors for medication Non-adherence in older adults with cognitive impairment who live alone. In J Geriatr Psychiatry 2012; 27(12): 1275-82.

24. Kardas P. Prevalence of non-adherence to medication among patients treated for selected chronic conditions. [Article in Polish]. Pol Merkur Lekarski 2008; 31(184): 215-220.

25. Hutchinson LC, Jones SK, West DS, et al. Assessment of medication management by community-living elderly persons with two standardized assessment tools: a cross-sectional study. Am J Geriatr Pharmacother 2006; 4(2): 144-153.

Received: 16/11/2013

Accepted in revised form: 15/04/2014

\section{Corresponding author:}

Jan Lužný, Department of Psychogeriatry, Mental Hospital Kroměříž, Havlíčkova 1265, Kroměříž, Czech Republic; e-mail: luznyj@plkm.cz 